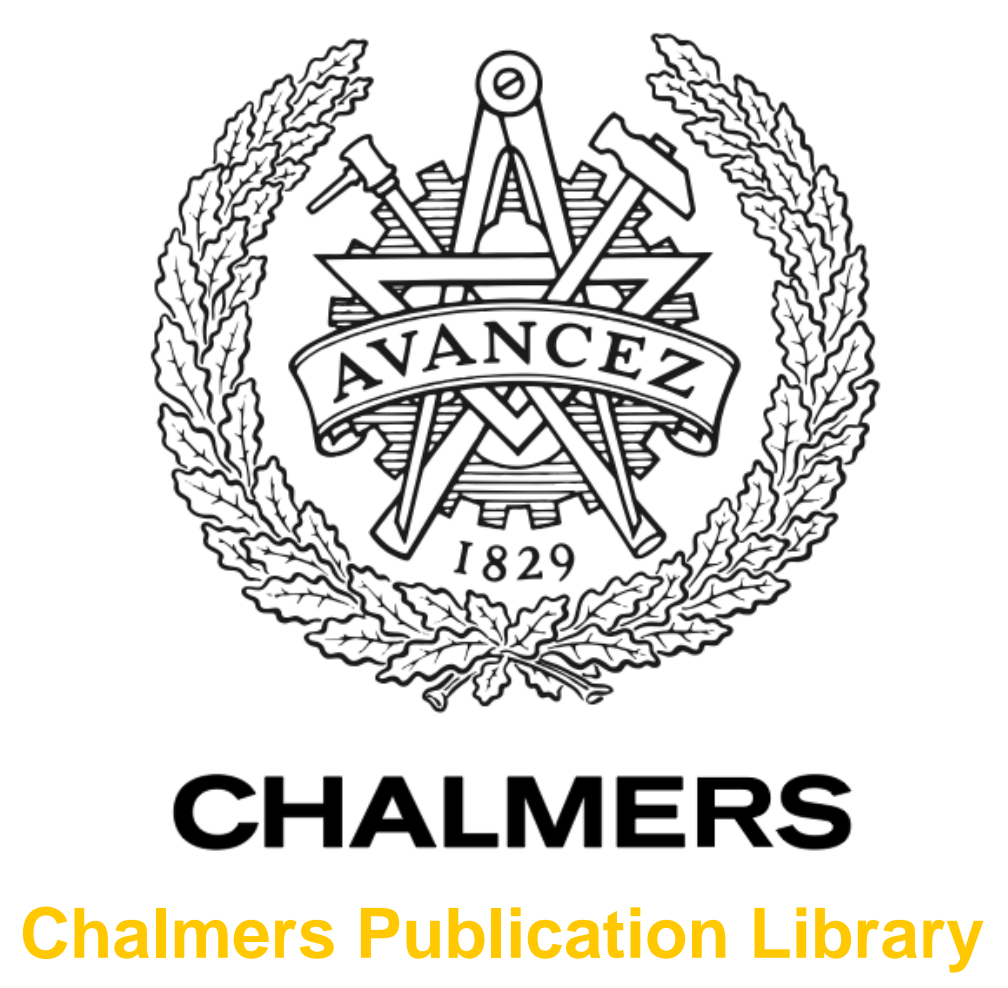

\title{
Why and How Traffic Safety Cultures Matter when Designing Advisory Traffic Information Systems
}

This document has been downloaded from Chalmers Publication Library (CPL). It is the author's version of a work that was accepted for publication in:

34th Annual Chi Conference on Human Factors in Computing Systems, Chi 2016

\author{
Citation for the published paper: \\ Wang, M. ; Lyckvi, S. ; Chen, F. (2016) "Why and How Traffic Safety Cultures Matter when \\ Designing Advisory Traffic Information Systems". 34th Annual Chi Conference on Human \\ Factors in Computing Systems, Chi 2016 pp. 2808-2818.
}

http://dx.doi.org/10.1145/2858036.2858467

Downloaded from: http://publications.lib.chalmers.se/publication/242512

Notice: Changes introduced as a result of publishing processes such as copy-editing and formatting may not be reflected in this document. For a definitive version of this work, please refer to the published source. Please note that access to the published version might require a subscription. 


\title{
Why and How Traffic Safety Cultures Matter when Designing Advisory Traffic Information Systems
}

\author{
MinJuan Wang, Sus Lundgren Lyckvi and Fang Chen \\ Interaction Design, Department of Applied IT, \\ Chalmers University of Technology \\ Gothenburg, Sweden \\ minw@chalmers.se \\ sus.lyckvi@chalmers.se fang.chen@chalmers.se
}

\begin{abstract}
With an increased number of both cars and drivers in the world, it is of great importance to design well-functioning driver support systems for them, in order to reduce the number of accidents. Despite the fact that the growing markets can be found in Asia, most advisory traffic information systems (ATIS) are designed for, and adapted to, the western market, and its predominant traffic safety cultures (TSCs). However, traffic safety cultures differ between different parts of the world, and this in turn affects how drivers respond to advisory traffic information. In our study, we designed an ATIS to accommodate two different traffic safety cultures. Our findings show that although drivers belonging to both TSCs drove more safely with our ATIS than without, they still responded very differently to it, using it to support their different driving strategies. This implies that the traffic safety culture of the driver cannot be ignored; ATIS designers need to study and understand the TSC they are designing for.
\end{abstract}

\section{Author Keywords}

Advisory Traffic Information System; traffic safety culture; traffic information; Vehicles; interaction design; information visualization;

\section{ACM Classification Keywords}

H.5.2 [Information interfaces and presentation]: User Interfaces.

\section{INTRODUCTION}

We are now in the age of "automated and cooperative driving", as stated by Bengler et al. [3]. Due to new safety regulations, environmental interests, new and better sensor technology and increased computational power, today's vehicles can be equipped with sophisticated technology. Using sensors and algorithms together with Vehicle-to-

Permission to make digital or hard copies of all or part of this work for personal or classroom use is granted without fee provided that copies are not made or distributed for profit or commercial advantage and that copies bear this notice and the full citation on the first page. Copyrights for components of this work owned by others than the author(s) must be honored. Abstracting with credit is permitted. To copy otherwise, or republish, to post on servers or to redistribute to lists, requires prior specific permission and/or a fee. Request permissions from Permissions@acm.org. CHI'16, May 07 - 12, 2016, San Jose, CA, USA

Copyright is held by the owner/author(s). Publication rights licensed to ACM.

ACM 978-1-4503-3362-7/16/05 ..\$15.00

DOI: http://dx.doi.org/10.1145/2858036.2858467
Vehicle and Vehicle-to-Infrastructure communication technologies, a car can detect, monitor and at least theoretically react automatically to every object around it. Consequently, Advanced Driver Assistance Systems (ADAS) are becoming increasingly common. Examples include adaptive cruise control, parking assistance, forward collision warning and lane departure warning.

As a consequence, the HMI design for in-vehicle information systems is very different from earlier decades [3]. The early ADAS focused on warnings only, which, from a design point of view, means presenting only the most relevant information in a very clear way to support rapid decision making on an operational level. On this level, most humans will respond in a similar way, since these situations boil down to immediate responses on a crisis, as well as inherent cognitive functions of the human brain. In the next step, ADAS were shifting from warning drivers towards supporting them with status information. These particular forms of ADAS are called Advisory Traffic Information Systems (ATIS), and they support decision making on a longer time scale, i.e. on the tactical and strategical levels, as opposed to on the operational level.

When moving from immediate/operational responses to tactical responses, culture in the form of traffic safety culture (TSC) comes into play in relation to both information needs and graphic design. Given the new sensor technology, we can in theory show very much information, so one salient issue is to decide how much information to show in an advisory system, and when and how. This in turn rises questions regarding what drivers need to know, what they want to know (which is not necessarily the same thing) and how to balance this in design without creating a cognitive overload. In deciding this, we need to consider traffic safety cultures, both in relation to driving safely and what that entails, as well as cultural connotations to colors and symbols. Moreover, different TSCs shape different behaviors (and vice versa), and it is worth discussing the notion of safe driving in relation to this.

Addressing some of the above issues, we have conducted a study to explore firstly whether it is possible to design a successful interface for drivers from more than one traffic safety culture, and secondly to which extent traffic safety cultures affect the use of this system. 
Based on our previous studies regarding Swedish vs Chinese drivers' information needs in various traffic situations, an interface was designed accordingly in an iterative process $[25,26]$. Using the findings from these studies, we designed the next version of this system, an ATIS that provides information regarding surrounding road users, in a way that accommodate both Swedish and Chinese drivers. In the coming pages we describe our evaluation of this system in the form of method, results, and the conclusions we have drawn; both regarding the design of ATIS in general, and on designing for different traffic safety cultures.

\section{BACKGROUND}

Traffic safety culture (TSC) has been defined [7] as "...the assembly of underlying assumptions, beliefs, values and attitudes shared by members of a community, which interact with a community's structures and systems to influence road safety related behaviors." This definition addresses that TSCs consist of common practices, expectations, attitudes, and informal or hidden rules that drivers learn from society regulations and by observing others in their communities. Those expectations, attitudes and values in turn motivate driving behaviors and strategies [15].

Whilst advocating the goal of improving safety by changing the traffic safety culture, Allen and Mercer [1] suggested that it was important to first understand the existing culture. Following this, a number of researchers have studied differences in traffic incidents and fatalities between national cultures. Lund and Rundmo [16] identified differences in risk perception and attitudes towards traffic safety and risk between Norwegian and Ghanaian drivers, finding that there were major differences between nations in perception of risks. In a similar vein, Ozkan et al [21] have concluded that cross-cultural differences exist in driving skills, after having compared drivers self-assessed skills with accident data and fatality rates in six different European countries.

On a more overarching level, the works by Hofstede provide a useful approach to understanding national cultural influences on behavior $[9,10]$. The six key cultural dimensions: individualism-collectivism, power distance, uncertainty avoidance, masculinity, indulgence and longterm-orientation, explain many observed differences in work behaviors between nations. When looking at traffic Safety cultures, we suspect that a few other dimensions could be relevant, for instance, traffic density, and, as the abovementioned studies show, drivers' perception of risks, which in turn is coupled to driving experience etc. Regardless of the specific cultural factors which may influence road safety-related behaviors, the research of Hofstede demonstrates the importance of nationally-shared beliefs, attitudes and values, for behavior.

As for Sweden vs. China, the two countries in our study, Hofstede' cultural studies show that the Swedish and Chinese cultures are differ quite substantially in five of Hofstede's six dimensions, only being similar in Uncertanty Avoidance. [10].

\section{Traffic in China vs Sweden}

In our study, we have been comparing Swedish and Chinese drivers since these represent to very different traffic safety cultures and also happen to be a Western vs an Asian market.

In China the automotive industry started booming in the 1990 s and has been gaining momentum since. Its total number of motor vehicles increased from 1.59 million in 1978 to over 120.89 million by the end of 2012[18]. It is one of the largest automotive markets in the world. From 2003 to 2012, the yearly increase of registered car numbers in China has been over $20 \%$. This however also means that in China, the roads are going to be much more crowded every year, and as a result, drivers in China are going to face more and more congested and dangerous traffic conditions. As a result, Advanced Driver Assistance Systems (ADAS) - of which ATIS are a part - are in demand in order to assist drivers with safe driving as well as easy driving. The technology for ADAS system is mature and many systems have become a standard system for the cars in China, as well as in European market.

Even though Swedish and Chinese traffic regulations are similar, driver behaviors are highly culturally mediated [14]. Compared to Western countries, the traffic environment in China is much more complex. In China, the road and traffic design among different cities can be very different as well [5]. Big cities like Beijing have many big roads with several parallel lanes in one direction; still many streets are often filled with pedestrians and bicycles, and in addition traffic signs are often perplexing. Many drivers in China usually have poor knowledge of traffic safety rules and show little respect for such rules. A field observation study in Beijing reported that only 63.6 percent of the observed drivers used

\begin{tabular}{lll}
\hline & Sweden & China \\
\hline Personal car driving history & Over 100 years & Only 20 to 30 years \\
Traffic density & Low & High \\
Traffic safety & Very good & Relatively bad \\
Attitude towards traffic regulations & Respectful, obedient & Disrespectful \\
Traffic situation & $\begin{array}{l}\text { Divided; different types of road users often } \\
\text { have own lanes, sidewalks etc. }\end{array}$ & The traffic is often mixed. \\
\hline
\end{tabular}

Table 1. How the traffic- related conditions differ between Sweden and China. 
a safety belt, just 40.4 percent signaled before changing lanes, and less than 1 percent turned on their headlights in light rain or moderate snow [30].

When studying fatal car-related accidents in China [31], it was found that the underlying causing factors were excessive speed $(14.0 \%)$, careless driving $(12.5 \%)$, driving without a license $(7.6 \%)$, driving in the wrong lane $(5.2 \%)$, drunk driving $(3.9 \%)$, other violations $(21.3 \%)$, and other behaviors affecting safety (27.3\%). Other studies [27] have also shown that the major factor contributing to the accidents was violations of traffic regulations were due to lack of safety awareness.

In comparison with China, Sweden benefits from over a 100 years of driving history, and is one of the safest countries regarding road transportation. In 2011, only 314 persons died and 4500 were severely injured [24]. Safety awareness among road users and transportation system design parties in Sweden is very high; "vision zero" - i.e. no fatal traffic incidents - has been set as a national goal for 2020 [2]. Further differences between Sweden and China can be found in Table 1.

In conclusion, these two countries are highly different in both what the driver population is like, how infrastructure looks and in general attitude to traffic safety.

\section{RELATED WORK}

Driving behavior is highly environmentally and culturally mediated. Hence, HMI design for Western drivers may not necessarily be optimal in other markets. Lindgren has for instance pointed out that a system considered useful in one country can be seen as almost worthless (or even harmful) in another one, and system settings feasible in one part of the world may not be suitable elsewhere [14].

\section{Cultural Differences and Attitudes Towards Advanced Driver Assistance Systems (ADAS)}

The differences of driving patterns between cultures are extreme in multiple dimensions. Lesch [12] made a crosscultural comparison of perceived hazard in response to warning components (colors, signal words and symbols) and warning configurations between product-users from China and US. They found that US participants consistently provided higher ratings of perceived hazard than Chinese participants. The differences in perception could result in a failure of product-users' relationship in taking appropriate precautions if it is assumed that perceived hazard-level must attain some threshold value in order to trigger compliance.

When it comes to ADAS, further differences between countries and traffic safety cultures have been found. According to a study by Lindgren et al[14], Chinese drivers dislike systems such as Driver Fatigue Warning, Forwardcollision warning, Lane-keeping and Blind Spot Monitoring, which indicates that their attitude towards different ADAS is very different from those of Western drivers. One suspicion is that in many cases warning signals signal too often in the dense traffic in China, and that Western designs are not in line with Chinese driving habits. When the system does not behave as expected, the driver tends to either abuse or reject the system entirely.

Similarly, Lian [6] conducted a study by interviewing drivers from Sweden and China respectively, and found that the preference of ADAS varied between the two driver groups. Chinese drivers preferred Adaptive Cruise Control and Backup Monitor, while Swedish drivers were more positive towards Lane Departure Warning and Forward Collision Warning. In another study, Young [28,29] compared regional differences in In-vehicle Information Systems (IVIS) design needs and preferences across drivers from Australia, U.S and China to determine the impact of any differences on IVIS design. The results of their studies demonstrate that introducing Western-style IVIS into China with little or no adaptation to meet the requirements and cultural preferences of this region is unlikely to be successful. It is known that the differences between regions such as culture, environment and traffic context can influence the needs, usability and acceptance of advisory systems. An in-vehicle system that works very well and of great value to drivers in one country may be of less value to those in another.

\section{Current findings on the Design of Advisory Traffic Information Systems (ATIS)}

As for designing ATIS (which are a sub-category of ADAS), there are a few studies, albeit not related to traffic safety cultures.

Summala [23], states that a driver's goal is to drive without discomfort and stay within his or her comfort zone. If the comfort zone boundary is exceeded and the safety margin violated, the driver has to respond to this by performing corrective actions. To maintain the comfort zone then, drivers need information from the surrounding traffic environment, which makes it possible for them to foresee potential hazards in good time; and this is also the main goal for our ATIS.

In a study conducted by Lindgren [13], an integrated advisory information display was compared with a display providing only critical warnings. The results showed that drivers kept a longer and thus safer distance to cars in front of them, when given advisory information rather than critical warnings only. In another study by Stanton [22], standard brake light displays were compared with a graded deceleration display expressing how hard the driver in front was braking. The results showed that the graded system produced more accurate behavioral responses from the behind driver during deceleration than the standard brake light display. Fagerlönn et al have done a similar study concerning sounds[8]. They compared three strategies of early advisory warnings in comparison with critical warnings only. Again, the results revealed that an early auditory signal more successfully notified the driver, than the warnings did. In addition, drivers had relatively few inappropriate responses or misses with advisory signals. 
Addressing time scales, Naujoks has carried out a series of studies $[19,20]$ that explored the effectiveness of advisory information in different timing scales and information specificity. The results underlined that early advisory information has more positive effects on driver performance than late warnings when surprising situations occurred

These studies indicate that the concept of giving advisory traffic information (as opposed to warnings only) is a promising approach to increase driving safety and comfort. However, most of the studies in literatures have focused the design of the signals per se. As for designing ATIS for the Chinese market specifically, studies are scarce [5].

\section{In Conclusion}

To conclude, there is a lack of deep understanding regarding the design of cross-regional interfaces; there are for instance no specific design guidelines in regard to this. Whereas we do have statistic data on traffic safety, or subjective data regarding drivers' information preferences, there was up until now no study concerning how drivers from different traffic safety cultures actually do respond - in terms of behaviors and safety - to the same ADAS.

\section{RESEARCH AGENDA}

This particular study is part of a longer endeavor to explore cross-regional design. It is preceded by three other studies.

In the first study [26], which centered on audio warnings, we also made a focus group study exploring traffic safety culture and information requirements Naturalistic driving films from both Sweden and China were used as a basis for discussion. The results showed us that in general Swedish and Chinese drivers have similar information requirements when they only interact with single road users. But, when the traffic situation gets complex and multiple road users are involved, the two categories had different information preferences. Swedish drivers required more information from frontal areas, whereas Chinese drivers preferred information presenting incoming traffic from the sides and from behind.

In our second study [17] we developed a visual ATIS using an iterative process. The ATIS aimed to give advisory information regarding surrounding road users. Two interfaces were developed; the first displayed only the directional information of road users around the car, while the second presented direction as well as the type of road users. Our findings indicated that the second version, showing both direction and user type, was preferred. However, the designs were tested only in Sweden, by Swedish drivers.

Thus, in our third study [25] we duplicated the experiment with the exact same experimental settings in China. The aim was not only to explore their response to the two designs, but also to inquire more about their respective information needs. Again, we found that the second design worked better in terms of reducing collision rates etc., but in addition we got additional feedback on the design per se, e.g. color, look and feel of icons etc. As for information needs, this comparative study showed (again) that Swedish drivers were most interested in three regions: Front Right, Front Left and Back Center. In contrast, Chinese drivers appreciated more information on Back Center, Back right and Back Left regions.

\section{RESEARCH AIM}

The findings of this last study in particular, helped us redesign the ATIS once more. The new, cross-cultural version was tested in both Sweden and China, as we set out to answer the following two questions:

1) Is it possible to design an interface for drivers from more than one traffic safety culture, making them drive more safely?

2) Will they respond in the same way to different traffic situations and to the information given by the ATIS?

It was our hypothesis that our ATIS would accommodate both traffic safety cultures, but that drivers' still would react differently to different situations.

\section{METHOD}

The study utilized a 2 × 2 × 4 mixed subjects design, having country (Sweden/China), treatment conditions (without ATIS / with ATIS), and traffic incidents scenarios (see below) as independent variables. It was first carried out in Sweden and later duplicated in China.

\section{Participants}

In all, there were 46 participants taking part, 24 from China and 22 from Sweden, as displayed in Table 2. Due to some technical issues as well as simulator sickness, data from 2 Swedish and 2 Chinese participants was not included in the data analysis.

The participants were recruited in the same way in both countries. Recruitment ads were posted in two very similar areas (science parks in urban areas). This choice was deliberate; we wanted drivers from the segment of people whose income is above average and thus could afford a middle class car. Although the two cohorts represented two rather different Traffic Safety Cultures, the participants were quite similar in other aspects: more than $77 \%$ of them have a higher degree than bachelor, and live and drive in urban areas with comparably dense traffic.

In this context it's also worth noting that whereas the Chinese participants only had an average of 4,4 years with a drivers' license (as opposed to 14,1 years for the Swedes), they however drove more often than their Swedish counterparts,

\begin{tabular}{llll}
\hline Nationality & $\begin{array}{l}\text { Mean Age } \\
(\boldsymbol{S D})\end{array}$ & $\begin{array}{l}\text { Gender } \\
(\mathbf{M} / \mathbf{F})\end{array}$ & $\begin{array}{l}\text { Mean Years with } \\
\text { Driving License }(\boldsymbol{S D})\end{array}$ \\
\hline Swedish & $34.6(13.9)$ & $16 / 6$ & $14.1(13.7)$ \\
Chinese & $31.0(8.6)$ & $20 / 4$ & $4.39(5.1)$ \\
\hline
\end{tabular}

Table 2: Participants in the study by nationality, age, gender and driving experience 


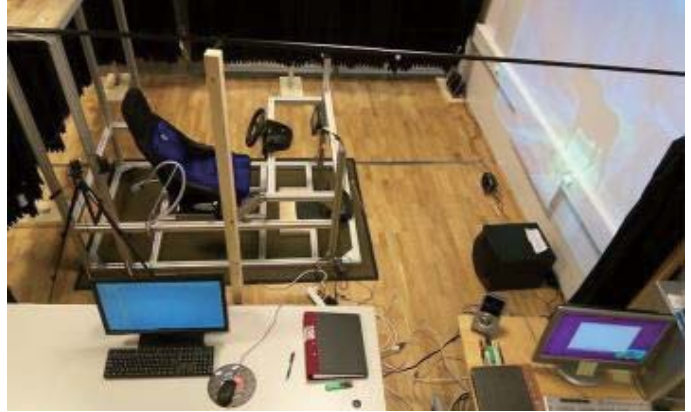

Figure1. Simulation setup

meaning that the two cohorts were more similar in terms of driving experience, than those particular numbers suggest. Studies suggest that after having driven 1000 miles a person's skill of foreseeing potential hazards is on par with more experienced drivers [11].

\section{Technical Setup}

In this experiment, one PC running STISIM Drive ${ }^{\circledR}$ software was utilized as driver simulator. A Logitech G25 Racing Wheel, which included pedals and a gearbox was installed. Another PC ran MATLAB to receive and analyze data from the simulator PC. A generic 7" Widescreen TFT LCD monitor was positioned above the steering wheel, to display the advisory traffic information system. A HD projector was used to project the simulated drive scenarios on the front wall. Two webcams were installed to record what the drivers saw on the road, as well as and their reactions to the incidents, e.g. steering, braking. This video data was synchronized with drivers' simulation data to better allocate the starting point of drivers' reactions to the incidents. Lastly, curtains created an enclosed area around the drivers, to allow them to feel more comfortable and not feel that they were being watched throughout the study. Figure 1 shows the layout of the simulation set up.

\section{The Advisory Traffic Information System (ATIS)}

As described under Research Agenda, this particular ATIS had been designed as a result of previous studies [4]. In short it is based upon dividing the area around the car into eight zones, using a color change as follows: white outline orange field - red field. This indicate the closeness of the other road user, red being the closest. In addition, pedestrians and cyclists were further indicated with an icon, since these two categories are especially vulnerable in traffic (see Figure 2).

The activation of the different information levels is based on two different factors, depending on the position of the object indicated. If the object is in the same lane as the driver, the calculation is based on time to collision (TTC). If the object instead is in an adjacent lane, the calculation is based on distance to collision. This compromise was made due to the limitation of the simulator output. The thresholds had been tested in our previous studies; this was necessary since the perception of distance is different when driving in a really

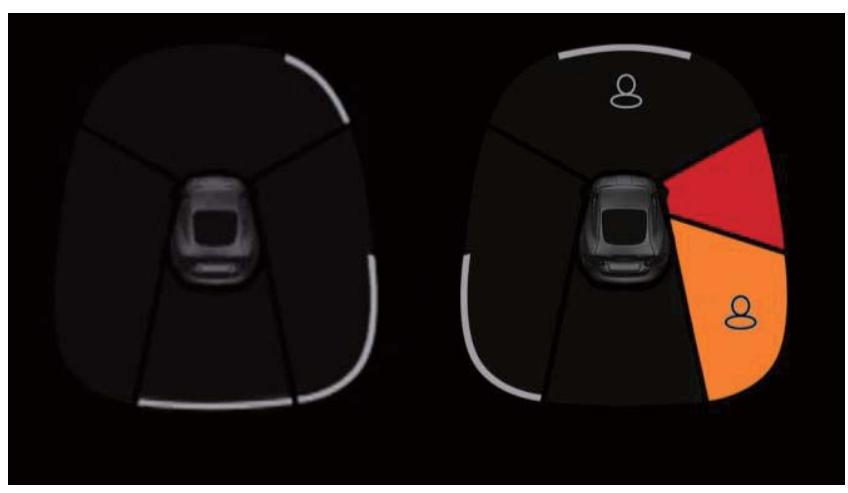

Figure 2. ATIS interface. To the left, three road users are at a distance (represented by the white lines) behind, to the back right and front right of the car. To the right, there's a pedestrian at a distance in front of the car, and another quite close in the right back blind spot. Moreover some sort of vehicle is very close to the car's right side.

\begin{tabular}{lll}
\hline Level & $\begin{array}{l}\text { Time to } \\
\text { Collision }\end{array}$ & $\begin{array}{l}\text { Distance to } \\
\text { Collision }\end{array}$ \\
\hline Informative (white) & $>6 \&<9 \mathrm{~s}$ & $<4.5 \mathrm{~m}$ \\
\hline Advisory (orange) & 3 to $6 \mathrm{~s}$ & $<3.5 \mathrm{~m}$ \\
\hline Critical (red) & $<3 \mathrm{~s}$ & $<2.5 \mathrm{~m}$ \\
\hline
\end{tabular}

Table 3. Thresholds for warnings in terms of time to collision (seconds) or distance to collision (meters).

car vs in the simulator, and thus we adapted the thresholds accordingly. Lastly, the TTC and distance to collision was translated into a color using the following thresholds, as seen in Table 3.

\section{The Traffic Incidents Scenarios Studied}

In order to design test incidents scenarios that represents the real traffic situations in both countries, the research team had observed over 100 naturalistic driving videos from both Sweden and China. These provided an understanding of the everyday traffic situations in both countries. In our design, we chose scenarios that covered common critical situations in both counties. These situations were chosen since it is during these the use of ATIS comes in play. Due to the higher traffic density in Chinese urban areas, they have a higher ratio of critical incidents but of course these and similar incidents occur often enough in Sweden as well.

To test the effects of the design, we chose two scenarios involving vehicles, and two involving pedestrians, i.e. four scenarios in total. However we added another five scenarios to the test drives in order to reduce learning effects between test drives under different conditions (without vs with ATIS). The order of the scenarios was randomized for each new test drive. Below we will describe the four scenarios we actually studied. The car (a) represents the driver's car. 


\section{Cut In}

In this event, car b (parked on the sidewalk on the right) attempts to start up and cut into the driver's driving lane, then it sees the driver's car in the rear view mirror, so it steers back to the sidewalk. On the lane to the left of car a (the driver's car), there are another two cars; one is in the

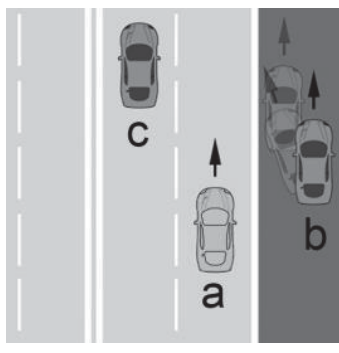
front and the other one is in the blind spot. Hence, changing lanes is not a good strategy. The considered safe approach in this scenario is to brake or steer slightly to left.

\section{Red Cab}

Here, two slow cars (b and c) drive in the left lane. Car b suddenly cuts into the driver's lane very fast directly in front of the driver's car (car a). At this moment, the other car (c) is in the blind spot of car a. In this scenario, the considered safe approach is to decelerate in order to avoid an accident.

\section{Intersection}

Here, the driver (in car a), has a green light. However, two pedestrians start crossing the street right in front of the driver anyway. One pedestrian is walking from right to left quite slowly, whereas the other is walking from left to right relatively fast. Of these two, the

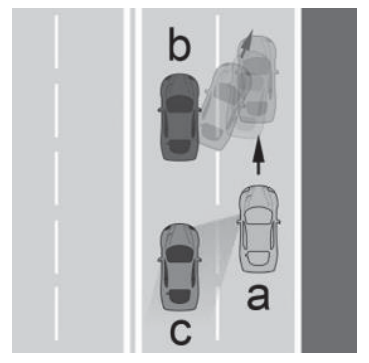
faster one starts walking first, and is partly hidden in the A pillar blind spot, whereas the slower one starts later. In this scenario, the considered safe behavior is to brake and wait for the pedestrian to pass

\section{Pedestrian Crossing}

Here, the road has narrowed down to a single lane in a residential area. A pedestrian suddenly walks out between the cars on the right side of the road. The driver (in car a) has a visual disadvantage in this scenario; visual contact with the pedestrian is made at a very short distance.

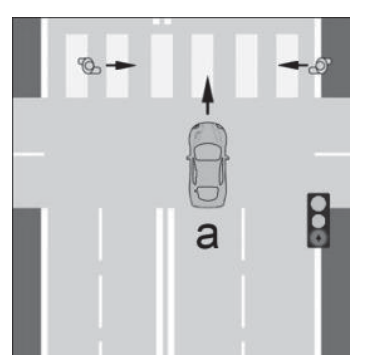
Therefore, the reaction time in this scenario is very short. Thus, the safest possible way to avoid the accident is to brake and steer at the same time.

\section{Overall Procedure}

Before the test drives, participants filled out a questionnaire regarding their driving style, as well as how they perceived the advisory traffic information system (ATIS) that was to be used (see below). The latter part aimed to collect usability assessments based on user's comprehension of the UI, and at the same time provide a learning session for users. For Swedish participants, the questionnaire was in English, for Chinese users in Chinese.

Thereafter, drivers were given instructions regarding the test drives. The researchers explained the purpose and the procedure of the experiment in general, and instructed drivers to keep the speed at $50 \mathrm{~km} / \mathrm{h}(31 \mathrm{mph})$, follow traffic regulations, stay in the right lane if possible etc. After getting some instructions on how to drive in the simulator, each subject could train as much as they wanted; when they were satisfied, the test started. Each subject drove twice, once with the ATIS and once without, in random order. Similarly, the order of the nine events that occurred in each test drive, was randomized for each new drive. After the test, participants were rewarded with two movie tickets.

\section{Measurements}

The measurements of driving performance that we used are the following dependent variables:

- Video observations of participants' simulation drive: these video observations were applied to doublecheck the participants' response point when they encountered the incidents.

- Brake throttle: Longitudinal acceleration due to the brakes (feet/second )

- Gas pedal: Longitudinal acceleration due to the throttle (feet/second)

- Steering wheel angle input: How much the steering wheel is turned, in the same or opposite direction (degrees).

- Number of collisions

- Maximum Steering wheel angle (SA): The difference between maximum and minimum steering angles when reacting to the event (degrees)

- Standard deviation of lateral position (SDLP): How the car has been moving sideways, thus reflecting the lane-keeping capability of drivers. The SDLP is calculated based on a historical time-series of lateral position. (meters)

- Distance to reaction (DR): The remaining distance to the incident when drivers first react (steer or brake) to the event. This reflects driver's safety consciousness regarding the situations. (meters)

- Standard deviation of speed (SDV): This reflects how the cars' speed changes from the events activate to the events finish. (feet/second)

\section{RESULTS}

Firstly, to understand, how the different TSC influenced driving behaviors, a driving pattern analysis was conducted based on how the participants' handled the different situations without the ATIS. Secondly, to investigate how drivers from different TSCs responded to the ATIS, a 2 


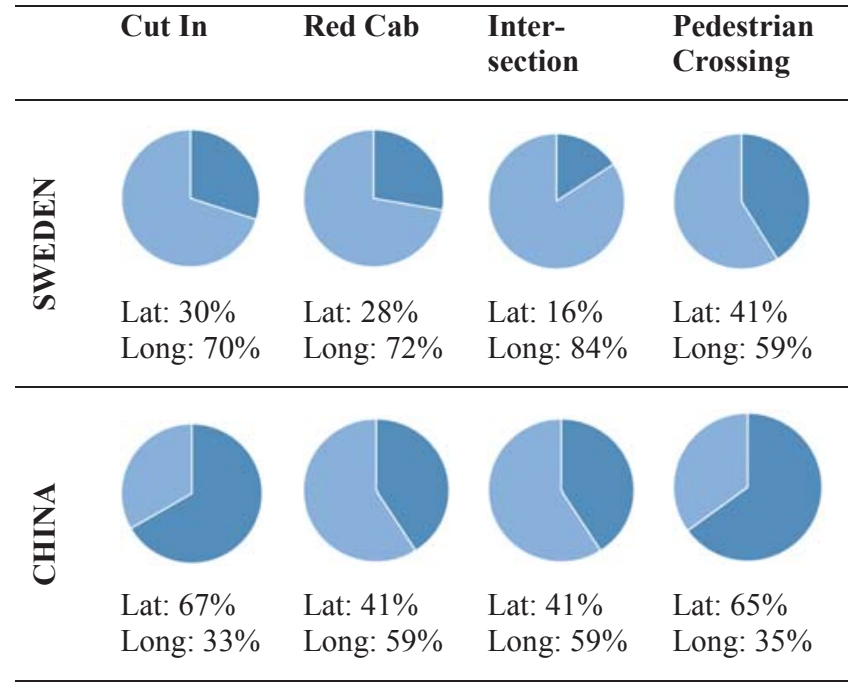

Table 4. How Swedish and Chinese driving behaviors differed in terms of longitudinal (increasing or decreasing speed) vs lateral (moving sideways, e.g. when changing lanes) movements.

(groups, between subject: country groups) x 2 (condition, within subjects: driving with/without ATIS) mixed-factor repeated-measures ANOVA was conducted for each dependent variable (measurement) in each scenario.

\section{Driving Patterns Without ATIS}

To each incident scenario, drivers could and did react differently. For example, the brake or gas throttles could be pressed or released, or the steering wheel could be turned, in any order. This data was collected from combined measurements analysis using data from the steering wheel, the gas pedal and the brake throttle input and video recording from test trials.

Overall, we can group these reactions into two distinct groups based on the drivers' first response reaction. Firstly, we have the behavior to first steer away from the situation before slowing down, as the driver intents to move laterally, e.g. change lanes. Secondly, we have the behavior to start with a decrease in speed (either by only releasing the gas throttle, or releasing it and then braking); these patterns display a longitudinal change.

The results showed that the driving patterns between Swedish and Chinese participants differed strongly, as can be seen in Table 4. Especially, when the situations involved pedestrians, the Swedish participants tended to brake or release the gas pedal as a means to stay out of a potentially dangerous situation; a strategy related to changing longitude. In comparison, the Chinese drivers were much more prone to steer first and then slow down or speed up, i.e. change lanes and move on: a latitudinal strategy. This is in line with previous findings on driving cultures and drive information requirements studies [26].

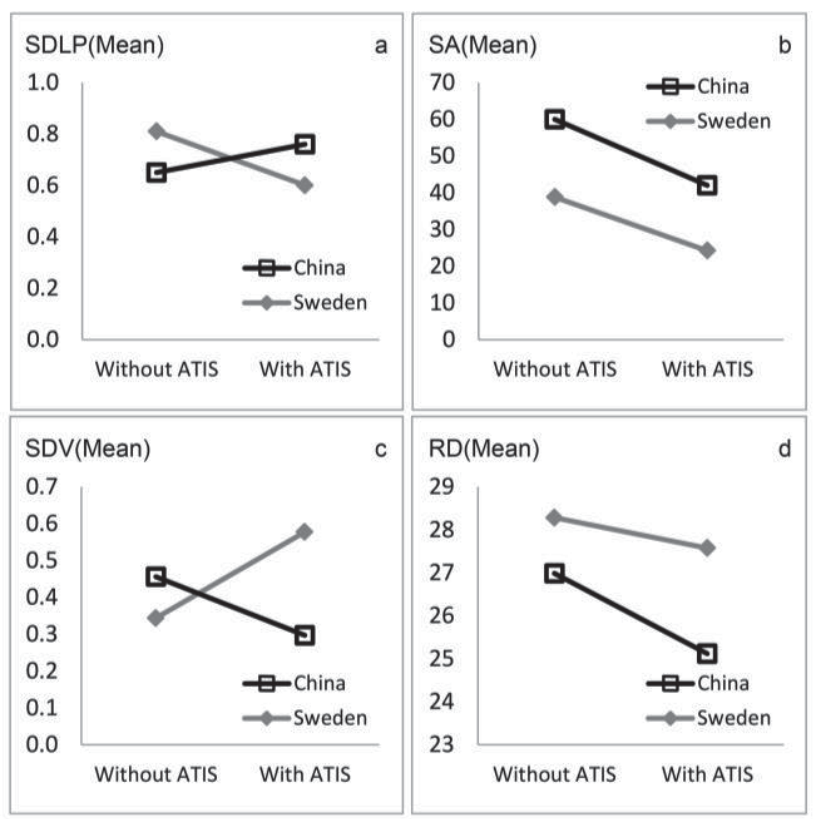

Figure 3. Mean of (a) standard deviation of lateral position, (b) maximum steering wheel angle, (c) standard deviation of speed and (d) distance to reaction in the $\boldsymbol{C u t}$ In scenario.

\section{Driving Patterns When Using The ATIS}

In this section we will describe the participants' behavior per scenario. We start with the comparison between using ATIS and not, and then compare the behavior of the two groups of participants.

Cut in

When driving with ATIS, both groups reduced their SA significantly $\left(\mathrm{F}(1,30)=10.01, \mathrm{P}=0.004, \eta_{\mathrm{p}}{ }^{2}=0.25\right)$. Swedish participants' SA was reduced around 12 degrees from 38.18 to 21.62 degree; Chinese participants gained the similar tendency: without ATIS $(M=62.93)$; with ATIS $(M=43.90)$.

In summary: When driving with ATIS, both groups steered less, which is a preferred and safer behavior, since veering too much in this particular scenario would potentially lead to a collision with the surrounding cars.

Interestingly, two interaction effects between condition and group were found in the measurements of SDLP $\left(\mathrm{F}(1,30)=4.98, \mathrm{P}=0.033, \eta_{\mathrm{p}}{ }^{2}=0.14\right)$ and $\operatorname{SDV}(\mathrm{F}(1,30)=7.68$, $\left.\mathrm{P}=0.009, \eta_{\mathrm{p}}^{2}=0.20\right)$. To understand the nature of the interaction effects further, we analyzed the interaction effects between condition and group by conducting a number of $\mathrm{T}$ tests to contrast the simple main effect of each group at each condition. However, no significant effects were found between groups and cross conditions. Therefore, these results indicated that the performance of two participates groups on SDLP and SDV are changing over conditions and they are changing significantly in different ways. In figure 3a and 3c, it shows that Swedish participants stabilized their lateral control when using the ATIS, however, in contrast, Chinese participants increased theirs. As for SDV, Swedish 
participants reduced speed variation with the ATIS, whereas Chinese participants increased their speed variation when they received the traffic information in advance.

No significant differences were found regarding Reaction Distance (RD), neither between groups or across conditions. Moreover, there were no accidents at all in this particular scenario.

In summary: The ATIS helped both groups towards driving more safely, but the results also showed that the groups changed their behavior in different ways when given information from ATIS. Swedish drivers veered less and drove more smoothly with the ATIS whereas Chinese drivers steered more, and also changed their speed more abruptly with the ATIS.

A possible explanation for this could be that when Swedish participants are informed about upcoming road users, they become aware of the hazard, they tend to slow down and not deviate sideways; basically the stay in their lane. As for Chinese drivers they perhaps react the opposite way; perceiving the hazard they look for an alternative way forward instead of braking.

\section{Red Cab}

In this scenario, the collision rates of Swedish participants showed that the ATIS helped to reduce the accidents from 9 to $6(33 \%)$.For the Chinese participants, the collision rates remained the same (12 accidents).

Statistically, the SDV did not change significantly for the Swedish participants, but there is a strong potential; without ATIS, SDV is 5.38 feet/s; with ATIS, SDV is 3.87 feet/s. i.e. the changes in speed were smoother with the ATIS.

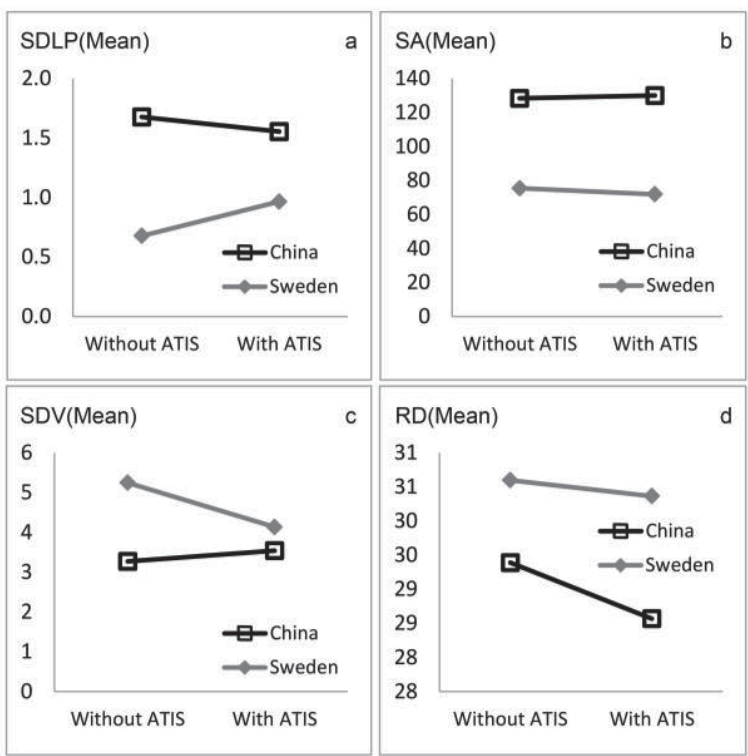

Figure 4. Mean of (a) standard deviation of lateral position, (b) maximum steering wheel angle, (c) standard deviation of speed and (d) distance to reaction in the Red Cab scenario.
In this scenario, four main effects between the groups were found (see Figure 4). Chinese participants had a larger SDLP $\left(\mathrm{F}(1,32) \quad=26.39, \quad \mathrm{p}<0.001, \quad \eta_{\mathrm{p}}{ }^{2}=0.45\right) \quad$ and $\quad \mathrm{SA}$ $\left(\mathrm{F}(1,32)=13.85, \mathrm{P}=0.001, \eta_{\mathrm{p}}{ }^{2}=0.30\right)$ than the Swedish. In comparison, the Swedish had a larger $\operatorname{SDV}(\mathrm{F}(1,32)=6.98$, $\mathrm{p}=0.013, \mathrm{\eta}_{\mathrm{p}}{ }^{2}=0.18$ ). The reaction distance also indicated that the Swedish participants had a greater safety margin than the Chinese drivers $\left(\mathrm{F}(1,33)=13.33, \mathrm{p}<0.001, \eta_{\mathrm{p}}{ }^{2}=0.288\right)$.

In summary: In this scenario, Chinese drivers were more prone to veer or change lanes, whereas Swedish drivers tended to slow down instead. The Swedish drivers drove more smoothly and had a greater safety margin than the Chinese; consequently they had fewer accidents that the Chinese without the ATIS and even fewer with the ATIS. For the Chinese the accident rate did not change. Thus the ATIS helped to improve the performance of the Swedish participants, but not the Chinese participants. Interestingly, the comparison between the two groups show that they differ in every aspect.

\section{Intersection}

In the intersection scenario, no main effects between conditions were observed. However, the Swedish participants using ATIS increased their brake distance with almost a half meter (without ATIS: brake distance $(\mathrm{M}=2,94$, $\mathrm{SD}=2,94)$ and with ATIS $(\mathrm{M}=3,27, \mathrm{SD}=0,83)$. Even though the result is not statistically significant, it shows a strong potential, and it is meaningful as the pedestrian is walking really close to the vehicle. In addition, the collision rates showed that Swedish participants (one collision per condition) drove much safer than Chinese participants (5 collision without ATIS; 6 collision with ATIS).

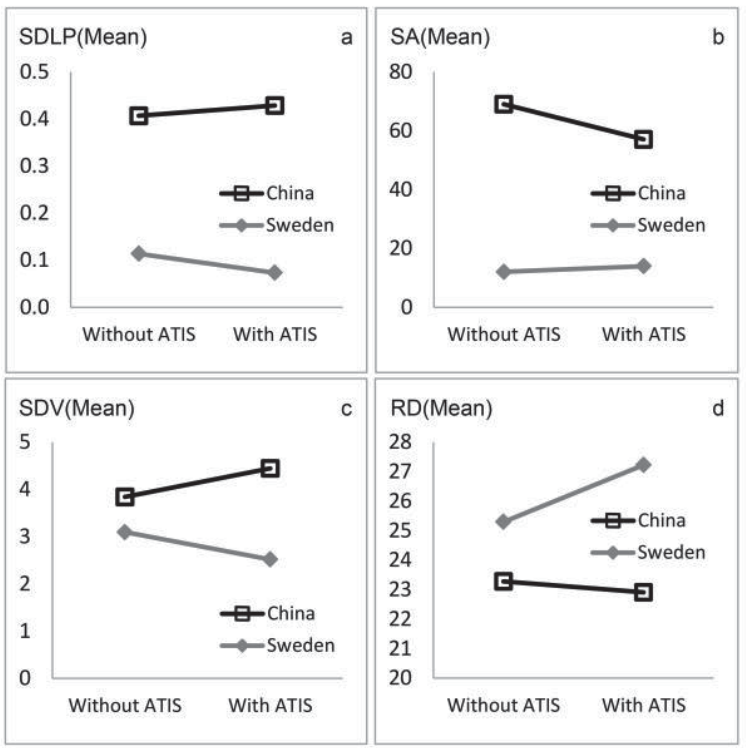

Figure 5. Mean of (a) standard deviation of lateral position, (b) maximum steering wheel angle, (c) standard deviation of speed and (d) distance to reaction in the Intersection scenario 
Remarkably, four main effects were found between the groups (see Figure 5). SDLP $(\mathrm{F}(1,26)=24.96, \mathrm{p}<0.001$, $\left.\eta_{\mathrm{p}}{ }^{2}=0.49\right), \quad \operatorname{SDV}\left(\mathrm{F}(1,26)=10.59, \mathrm{p}=0.003, \eta_{\mathrm{p}}{ }^{2}=0.29\right), \quad \mathrm{SA}$ $\left(\mathrm{F}(1,26)=27.30, \mathrm{p}<0.001, \eta_{\mathrm{p}}{ }^{2}=0.51\right)$ and $\mathrm{RD}(\mathrm{F}(1,32)=5.55$, $\left.\mathrm{p}=0.025, \eta_{\mathrm{p}}{ }^{2}=0.15\right)$.

There were no significant effects on driver performance with and without the ATIS. However, again, the two cohorts behaved very differently. The Chinese participants tended to veer more than the Swedish. Overall, Swedish participants handled the situation in advance and with a smaller speed variation; without the ATIS they reacted 2 meters earlier than the Chinese; with the ATIS this increased to 4 meters. As a result they also changed their speed more smoothly.

\section{Pedestrian crossing}

When driving with ATIS, both groups increased their SA significantly $\left(F(1,25)=5.04, p=0.034, \eta_{p}^{2}=0.17\right)$. Without ATIS (SE: $M=28.19 ; C N: M=69.02)$; With ATIS (SE: M= 35.12; $\mathrm{CN}: \mathrm{M}=110.99)$.

In this scenario, a pedestrian walks out on the street from between two cars, meaning that visual contact is established very late. The Swedish participants' mean reaction distance was 19.99 meters without the ATIS and 20.66 with. The Chinese participants' was 14.27 vs 15.66 meters. Since they drove with ca $50 \mathrm{~km} /$ hour, this translates to 1 to 1.5 second to respond. With such short reaction distance, the most suitable way to avoid the accident is to both steer and brake. A possible explanation is that when the ATIS points out the hidden pedestrian, they adjusted their steering wheel controls accordingly.

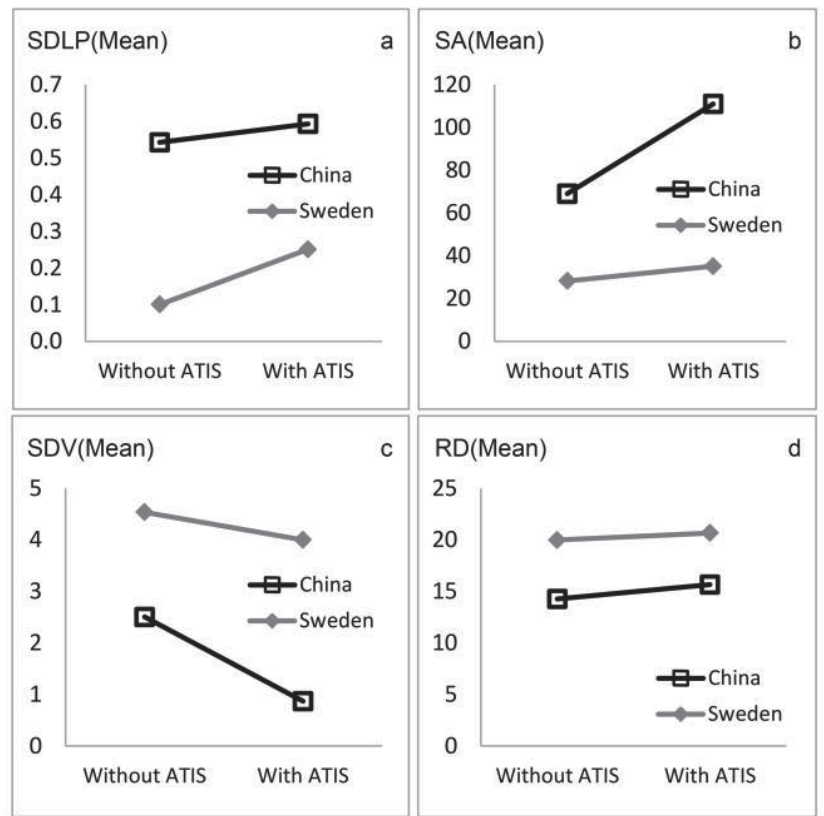

Figure 6. Mean of (a) standard deviation of lateral position, (b) maximum steering wheel angle, (c) standard deviation of speed and (d) distance to reaction in the Pedestrian Crossing scenario.
The ATIS also helped reduce the number of collisions; from 9 to 6 for Swedish drivers, and from 12 to 9 for Chinese drivers. Given that this scenario is very critical, the accident rates are comparative high in both groups.

There were 4 main effects observed between the groups (see Figure 6). $\mathrm{RD}\left(\mathrm{F}(1,33)=20.66, \mathrm{p}=0.012, \eta_{\mathrm{p}}{ }^{2}=0.18\right)$ and $\operatorname{SDV}\left(\mathrm{F}(1,25)=14.03, \mathrm{p}=0.001, \eta_{\mathrm{p}}{ }^{2}=0.36\right)$ showed that Swedish drivers had large reaction distance and speed change than Chinese drivers in both conditions. In comparison, Chinese participants had large lateral changes than Swedish participants in both condition from the results of SDLP $\left(\mathrm{F}(1,25)=7.53, \mathrm{p}=0.011, \eta_{\mathrm{p}}{ }^{2}=0.23\right)$ and $\mathrm{SA}(\mathrm{F}(1$, 25) $\left.=19.07, \mathrm{p}<0.001, \eta_{\mathrm{p}}{ }^{2}=0.43\right)$.

In summary: In this scenario the ATIS helped drivers drive more safely. However their behaviors very clearly show their different strategies. When meeting pedestrians, Swedish drivers tended to slow down or stop the car, as this reaction matches with the Swedish traffic law that vehicles should give way to pedestrians, while Chinese drivers tend to steer the car sideways to avoid a crash instead of slowing down.

\section{DISCUSSION}

As pointed out earlier, we set out to answer the following two questions:

1) Is it possible to design an interface for drivers from more than one traffic safety culture, making them drive more safely?

2) Will they respond in the same way to different traffic situations and to the information given by the ATIS?

As our results clearly show, both Chinese and Swedish participants drove more safely with the ATIS than without. As is also shown, they did however not respond in the same way to different traffic situations; we had suspected this would happen, but it was a surprise to us to see how the participants explicitly utilized the system. Below, we will discuss these findings further.

\section{How Drivers Utilized the ATIS}

Overall, we see the same driving behaviors reflected in our data on driving patterns. For the majority of Swedish participants the first reaction was a longitudinal strategy (i.e. slowing down), whereas Chinese participants' first response was steering. The significant differences found between groups indicate that even if performance improved with the ATIS, it did not change or shaped their original behaviors. Swedish participants maintained their longitudinal strategy whereas the Chinese preferred lateral strategies.

The Cut In scenario is especially interesting in this regard. Chinese participants' data indicate that the ATIS helped the drivers by making them aware of cut in vehicle. But instead of braking or stopping for the vehicle to pass first, they utilized the information from the ATIS, in that they instead of stopping, switched to another lane (which they through the ATIS knew was empty) to avoid the vehicle but still be able to drive on, despite the fact that this behavior is not 
encouraged from a safety perspective. So here, the interface actually encourages a lateral strategy for a driver preferring this.

This particular result is in line with an earlier observation we did in China with the Blind spot warning information. When the blind spot information system (BLIS) is activated, the Chinese drivers don't do any visual check up on the other lane, they just change lane based on the information showed on the BLIS. This is a typical non-intended use of the system, since the drivers trust the system too much. Most of the automated systems are reliable and usually work as intended, but unfortunately, some may incidentally fail or behave unpredictably. It is problematic if drivers trust them too much, i.e. rely uncritically on their cars' ADAS without recognizing their limitations or irregular behaviors.

In contrast, Swedish drivers used ATIS information as a basis for slowing down more than anything else; and examples can be found in the Intersection scenario, where Swedish drivers utilized the ATIS to react earlier than the Chinese since, as shown from the information requirements, Swedish drivers are more interested in information regarding what is going on in front of them.

TSCs Matter, And What Is Safe, Anyway?

Both driving performance measurement analysis and drive pattern category analysis indicated that when drivers interacted with pedestrians, Chinese drivers and Swedish drivers showed totally different behaviors. Chinese participants tended to steer away to avoid the pedestrians but keep on driving. Swedish drivers, on the other hand, slowed down, being more concerned about their safety distance to other road users. This reflects two things; firstly how drivers perceive the risk of causing an accident involving vulnerable road user. Secondly, it shows how drivers have been socialized into certain behaviors as per based on the traffic situations they are used to. Swedish drivers break, based on that the car behind, likely has enough safety margin to break too. Chinese drivers change lanes if possible, assuming that the cars behind do not have time to break, which might cause a serial crash.

In accordance, field studies on Chinese traffic situations and driver behaviors have pointed out that high traffic density, ignorance of traffic rules and chaotic combination of trucks, cars, scooters, bicycles and pedestrians are the main problems of driving in the city. Moreover, in China, it is not the driver's obligation to give way to the vulnerable road users, such as pedestrians - instead the other road users need to watch out for the cars. Therefore, when drivers encountered the situations with vulnerable road users, they tended to avoid them by changing to another lane and then continue driving. Consequently, the second most common crash pattern in China in 2009, contributing to road traffic deaths was side-to-side crash (25.5\%): only $1 \%$ less than frontal crash making these the two most common crash patterns [31].
This shows how notions of "safe behavior" may differ from case to case. If an emergency brake will lead to a serial collision, it might be better to change lanes in order to avoid a pedestrian, hopefully not hitting another, unnoticed pedestrian.

The unanticipated behavior described in the previous section, as well as our insights in the very different traffic situations in Sweden vs China raised a design question for us: is it up to us to assume that there is some international standard for what "safe driving" entails? Can we always know which behavior is safe and which not, i.e. can we design our systems in such a way that we shape driver behavior towards this assumed safe behavior? We are no longer certain that this is neither possible nor wise. Our study shows that Traffic Safety Culture is more deeply ingrained in the drivers' behaviors than we thought, and in conclusion we believe that one needs to think through this issue more than once before trying to design a more persuasive interface.

\section{CONCLUSION}

It was already known that Traffic Safety Cultures strongly affect how drivers respond to a situation; we have also shown how different Traffic Safety Cultures can result in different driving strategies. Moreover, although it is possible to design a cross-cultural interface, drivers from different TSCs will still "game" the system. This implies that ATIS designers need to study and understand the motives of these utilization, in order to refine the ATIS further and anticipate the potential misuses in respect to a given TSC.

\section{ACKNOWLEDGMENTS}

This project is a part of FFI ADAS presentation project. We would like to thank all our study participants, as well as Maria Kougioumoutzi, Calin Giubega and Peter Chen who designed the ATIS interface and conducted the study in Sweden. Thanks also to Liao Yuan at the Automotive Engineering Department, Tsinghua University in China for her support on data clinic and information source about Chinese traffics.

\section{REFERENCES}

1. Paul Allen and G. William Mercer. 2007. The role of public surveys in measuring program effectiveness and improving road safety. Improving Traffic Safety Culture in the United States, 93.

2. Matts-Åke Belin, Per Tillgren, and Evert Vedung. 2012. Vision Zero - a road safety policy innovation. International journal of injury control and safety promotion 19.2: 171-179.

3. Klaus Bengler, Klaus Dietmeyer, Berthold Färber, Markus Maurer, Christoph Stiller, and Hermann Winner. 2014. Three Decades of Driver Assistance Systems: Review and Future Perspectives. Intelligent Transportation Systems Magazine,IEEE 4, October: 6-22. 
4. Fang Chen, Călin Giubega, Peter Chen, and Minjuan Wang. 2014. Designing an In-Vehicle Advisory System to Support Traffic Awareness. 10th ITS European Congress, Helsinki, Finland, 10th ITS European Congress, 1-12.

5. Fang Chen, Minjuan Wang, Xi Chan, and Jiaqi Li. 2012. How Shall We Design the Future Vehicle for Chinese Market. Work: A Journal of Prevention, Assessment and Rehabilitation 41: 4265-4272.

6. Lian Duan. 2012. Culture Differences Analysis A Comparison between Chinese Drivers and Swedish Drivers on Driving Behaviors.

7. Jason Edwards, James Freeman, David Soole, and Barry Watson. 2014. A framework for conceptualising traffic safety culture. Transportation Research Part F: Traffic Psychology and Behaviour 26: 293-302.

8. Johan Fagerlönn, Stefan Lindberg, and Anna Sirkka. 2012. Graded auditory warnings during in-vehicle use: using sound to guide drivers without additional noise. Proceedings of the 4th International Conference on Automotive User Interfaces and Interactive Vehicular Applications, c: 85-90.

9. Geert Hofstede, Gert Jan Hofstede, and Michael Minkov. 1997. Cultures and organizations. New York, NY: McGraw Hill.

10. Geert Hoftede, Gert Jan Hofstede, and Michael Minkov. 2010. Cultures and organizations: software of the mind: intercultural cooperation and its importance for survival. McGraw-Hill.

11. Neale Kinnear, Steve W. Kelly, Stephen Stradling, and James Thomson. 2013. Understanding how drivers learn to anticipate risk on the road: A laboratory experiment of affective anticipation of road hazards. Accident Analysis and Prevention 50: 1025-1033.

12. Mary F Lesch, Pei-Luen Patrick Rau, Zhengxuan Zhao, and Chengyi Liu. 2009. A cross-cultural comparison of perceived hazard in response to warning components and configurations: US vs. China. Applied ergonomics 40, 5: 953-61.

13. Anders Lindgren, Alexander Angelelli, Paul Alvardo Mendoza, and Fang Chen. 2009. Driver behaviour when using an integrated advisory warning display for advanced driver assistance systems. IET Intelligent Transport Systems 3, 4: 390-399.

14. Anders Lindgren, Fang Chen, Patrick W Jordan, and Haixin Zhang. 2008. Requirements for the Design of Advanced Driver Assistance Systems - the differences between Swedish and Chinese drivers. International Journal of Design 2, 2: $41-$ 54.

15. Lawrence P Lonero. 2007. Finding the next cultural paradigm for road safety. In Improving traffic safety culture in the United States: The journey forward.

16. Ingunn Olea Lund and Torbjörn Rundmo. 2009. Crosscultural comparisons of traffic safety, risk perception, attitudes and behaviour. Safety Science 47, 4: 547-553.

17. David Marshall and Minjuan Wang. 2012. Development of an In-Vehicle User Interface for Traffic Information Presentation. Proceedings of the 44th Annual International Nordic Ergonomics and Human Factors Society Conference, Saltsjöbaden, Sweden.
18. National Bureau of Statistics of China. 2013. Statistical Communiqué of the People's Republic of China on the 2012 National Economic and Social Development. China.

19. Frederik Naujoks and Alexandra Neukum. 2014. Timing of in-vehicle advisory warnings based on cooperative perception. In Proceedings of the Human Factors and Ergonomics Society Europe Chapter Annual Meeting, 193206.

20. Frederik Naujoks and Alexandra Neukum. 2014. Specificity and timing of advisory warnings based on cooperative perception. In Mensch \& Computer-Workshopband: 14, 229 238.

21. Türker Ozkan, Timo Lajunen, Joannes El Chliaoutakis, Dianne Parker, and Heikki Summala. 2006. Cross-cultural differences in driving skills: a comparison of six countries. Accident; analysis and prevention 38, 5: 1011-8.

22. Nash Stanton, Roger Lew, Nolan Boyle, Brian P. Dyre Rowdy J. Hope, and Ernesto A. Bustamante. 2011. An Implementation of a Graded Deceleration Display in Brake Light Warning Systems. Proceedings of the Human Factors and Ergonomics Society Annual Meeting, 55, 1: 1573 1577.

23. H Summala. 2007. Towards understanding motivational and emotional factors in driver behaviour: Comfort through satisficing. In Modelling driver behaviour in automotive environments. Springer London, 189-207.

24. The Swedish Transport Administration. 2012. Analysis of Road Safety Trends 2011.

25. Min Juan Wang, Lian Duan, Jia Xin Wang, et al. 2016. Drive Advisory System : Will Swedish and Chinese Drivers Appreciate it in the Same Way? International Journal of Engineering and Technology 8, 4: 286.

26. MinJuan Wang, Yi Ci Li, and Fang Chen. 2012. How can we design 3D auditory interfaces which enhance traffic safety for Chinese drivers? Proceedings of the 4th International Conference on Automotive User Interfaces and Interactive Vehicular Applications, ACM Press, 77-83.

27. Cheng-qiu Xie and Dianne Parker. 2002. A social psychological approach to driving violations in two Chinese cities. Transportation Research Part F Traffic Psychology and Behaviour 5, 4: 293-308.

28. K. L. Young, Megan Bayly, and Michael G. Lenné. 2012. Cross-regional in-vehicle information system design: the preferences and comprehension of Australian, US and Chinese drivers. IET Intelligent Transport Systems 6, 1: 36.

29. Kristie L Young, Christina M Rudin-Brown, Michael G Lenné, and Amy R Williamson. 2012. The implications of cross-regional differences for the design of In-vehicle Information Systems: A comparison of Australian and Chinese drivers. Applied ergonomics 43, 3: 564-73.

30. Wei Zhang, Yueng-Hsiang Huang, Matthias Roetting, Ying Wang, and Hua Wei. 2006. Driver's views and behaviors about safety in China--what do they NOT know about driving? Accident; analysis and prevention 38, 1: 22-7.

31. Xujun Zhang, Hongyan Yao, Guoqing Hu, Mengjing Cui, Yue $\mathrm{Gu}$, and Huiyun Xiang. 2013. Basic characteristics of road traffic deaths in china. Iranian journal of public health 42,1 : $7-15$. 